Arq. Bras. Med. Vet. Zootec., v.66, n.6, p.1941-1945, 2014

\title{
Comunicação
}

[Communication]

\section{Identificação de loci microssatélites com potencial de amplificação na espécie de Peixe-Rei (Odontesthes humensis)}

[Identification of microsatellite loci with amplification potential in "pejerrey" (Odontesthes humensis)]

\author{
R.A. Tavares $^{1}$, S.R.N. Piedras ${ }^{2}$, M.D. Nunes ${ }^{1}$, D.B. Almeida ${ }^{1}$, C.G.A. Moreira ${ }^{3}$, J.M. Fernandes ${ }^{1}$, \\ S.F. Freitas ${ }^{2}$, H.L.M. Moreira ${ }^{3}$, J.L.O.F. Pouey ${ }^{2}$, N.J.L. Dionello ${ }^{2}$ \\ ${ }^{1}$ Programa de pós-graduação - Universidade Federal de Pelotas - UFPel - Pelotas, RS \\ ${ }^{2}$ Universidade Federal de Pelotas - UFPel - Pelotas, RS \\ ${ }^{3}$ Instituto de Biologia - Universidade Federal de Pelotas - UFPel - Pelotas, RS
}

Com os avanços da tecnologia de sequenciamento de próxima geração, recursos genômicos estão se expandindo rapidamente, mesmo para as espécies de peixes não modelo. Entre as espécies de peixes aquícolas, muitas economicamente importantes estão sendo sequenciadas, tais como Salmão do Atlântico (Salmo salar) (Lien et al., 2011) e Tilápia do Nilo (Oreochromis niloticus) (Guyon et al., 2012). Esses recursos genéticos incluem etiquetas de sequências expressas (EST), mapas físicos, mapas de ligação genéticos e marcadores moleculares.

O marcador molecular do tipo microssatélite (SSRs-Simple Sequence Repeats) é uma ferramenta satisfatoriamente utilizada em estudos de estrutura de populações, conservação de espécies e gestão de recursos genéticos (Tavares et al., 2011), por apresentar alto polimorfismo, codominância e herança simples. O desenvolvimento de marcadores microssatélites exigia um dispendioso esforço técnico, com procedimentos demorados e caros. Esses procedimentos incluem técnicas, como a criação de bibliotecas enriquecidas para loci SSRs, clonagem, hibridização para detectar clones positivos, isolamento de plasmídeo e sequenciamento (Beheregaray e Sunnucks, 2000; Barroso et al., 2003; Koshimizu et al., 2009). Entretanto avanços na tecnologia de sequenciamento de DNA vêm proporcionando métodos mais eficientes e de baixo custo para desenvolver marcadores moleculares para espécies que não possuem dados disponíveis, gerando os chamados loci potencialmente amplificáveis (PAL) (Castoe et al., 2012; Lance et al., 2013).

O peixe-rei é um representante da ordem atheriniformes, que inclui peixes encontrados em águas doces, marinhas, tropicais e temperadas de todo o mundo. O peixe-rei é um exemplar da subfamília atherinopsinae, que é composta por seis gêneros em dois grupos antitropicais: Atherinopsini na América do Norte (Atherinops, Atherinopsis, Colpichtys, Leuresthes) e Sorgentinini na América do Sul (Basilichthys, Odontesthes) (Dyer, 2006). Odontesthes é o gênero com a maioria das espécies, e mais amplamente distribuído, sendo encontrado em águas costeiras marinhas e lagoas de água doce da América do Sul. No Brasil, está presente principalmente nas lagoas Mirim e Mangueira, caracterizadas como um importante recurso pesqueiro, onde já foram descritas várias espécies, sendo $O$. humensis uma das mais comumente encontradas (Dyer, 2006).

O conhecimento sobre a variabilidade genética e os padrões de estrutura das populações são prérequisitos para o desenvolvimento de estratégias para aquicultura, através de futuros programas de melhoramento genético. No entanto, estudo de variabilidade genética em $O$. humensis requer o 
desenvolvimento de marcadores moleculares polimórficos.

Este estudo tem o objetivo de identificar loci potencialmente amplificáveis de microssatélites para a espécie de Peixe-Rei $O$. humensis, visando compreender a genética dessa espécie.

O material biológico utilizado neste estudo foi obtido de coletas na lagoa Mangueira (Santa Vitória do Palmar, Brasil - S 3259’12” e O $\left.52^{\circ} 42^{\prime} 42 ”\right)$. O DNA genômico total foi extraído a partir de nadadeira caudal (200-300 mg) de $O$. humensis, usando separação orgânica pelo protocolo modificado de sal (Barrero et al., 2008).

Uma única biblioteca genômica foi preparada a partir de 20ng de DNA, de acordo com o protocolo padrão do kit Illumina Nextera DNA Library (Illumina, San Diego, USA). O sequenciamento da biblioteca foi conduzido em um sequenciador GAIIx (Illumina, San Diego, USA) com leituras de 150 pares de bases. As leituras obtidas foram analisadas com 0 programa PAL_FINDER_v.0.02.03 (Castoe et al., 2012), identificando microssatélites com dinucleotídeos (2mer), trinucleotídeos (3mer), tetranucleotídeos (4mer), pentanucleotídeos (5mer) e hexanucleotídeos (6mer). Essas repetições deveriam ser simples e de pelo menos 12 pb de comprimento para 2mer, 3mer e 4mers, e de pelo menos três repetições para 5 mers ou 6mers. Após a identificação os loci SSRs foram agrupados para um subdiretório local do programa Primer3 v.2 (Lance et al., 2013) para o desenho dos primers.

Obtiveram-se 1.265.204 leituras, contendo aproximadamente $0,184 \mathrm{Gpb}$. O tamanho do genoma de $O$. humensis é desconhecido, embora genomas de peixes que foram pesquisados apresentam em média 1,250 Gbp (Katagiri et al., 2005; Jiang et al., 2013); sendo assim, a cobertura total foi de aproximadamente $15 \%$ do genoma.

Do total de leituras obtidas, 75.347 continham SSRs; no entanto, 34\% apresentaram sequências flanqueantes bastante adequadas para desenho de primers, produzindo $25.806 \mathrm{PAL}$, sendo 21\% de 2 mers, $22 \%$ de 3 mers, $37 \%$ de 4 mers, $13 \%$ de 5 mers e $7 \%$ de 6 mers (Fig. 1).

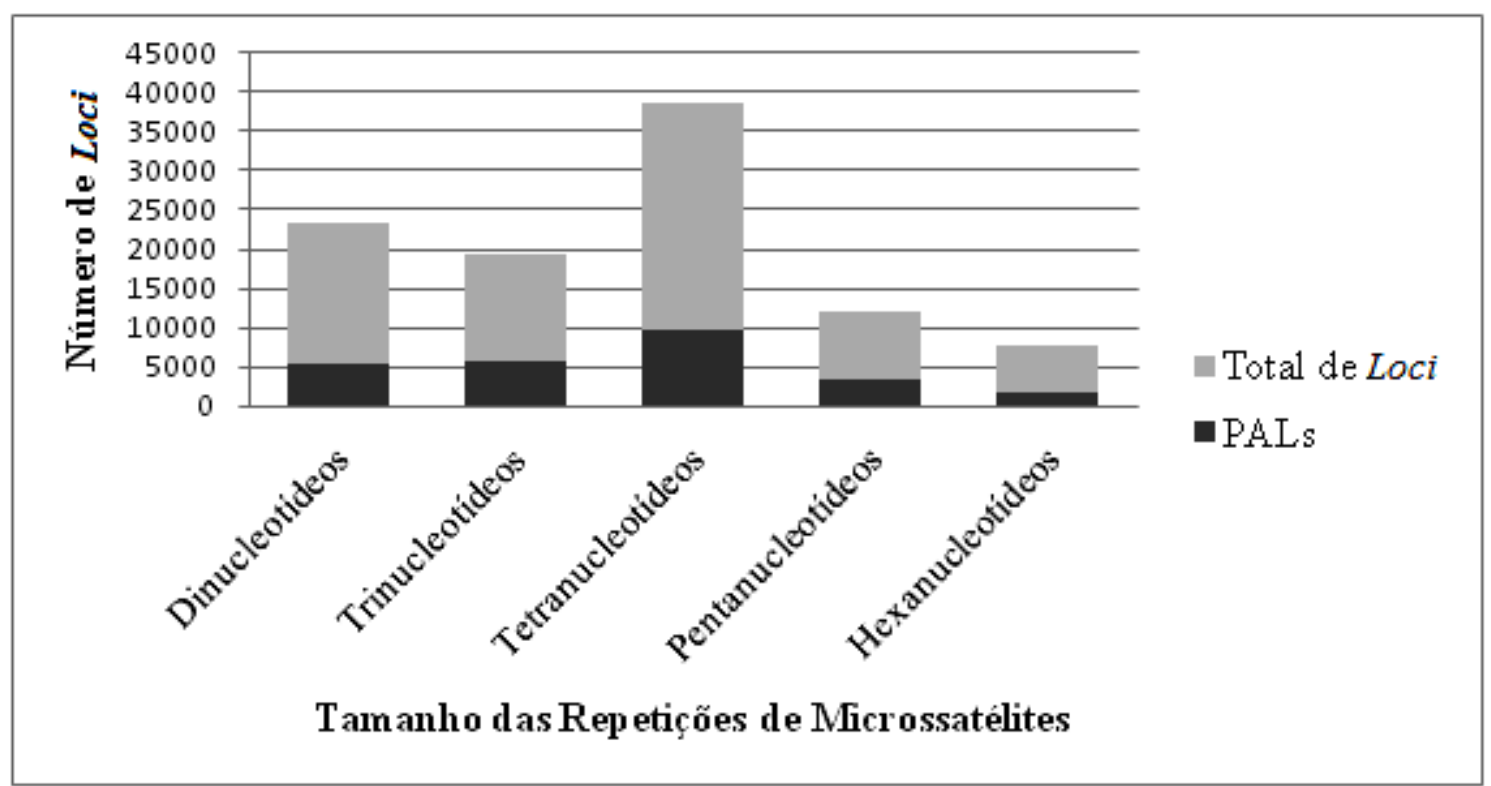

Figura 1. Comparação entre o número total de loci e o número de loci potencialmente amplificáveis (PALs), identificados por tamanho das repetições de microssatélites.

Em comparação com a espécie modelo “Zebrafish” (Danio rerio) (116.915 SSRs) (Rouchka, 2010), O. humensis apresenta números de loci microssatélites inferiores, porém, para obtenção dos loci SSRs, em "Zebrafish", foi utilizado o genoma total de 
aproximadamente $1,7 \mathrm{Gpb}$ e para este estudo, um total de $0,185 \mathrm{Gpb}$.

Para Carpa Siamês (Henichorynchus siamensis), 65.954 sequências foram obtidas com a plataforma 454 GS-FLX (Roche, Branford, USA), sendo que 1.837 eram SSRs (Iranawati et al., 2012). Lance et al. (2013) sequenciaram 0,5 Gpb do genoma de "Speckled Dace" (Rhinichthys osculus) e "Mountain Whitefish" (Prosopium williamsoni), apresentando em torno de 30.000 e 26.000 PALs, respectivamente. Essas divergências podem ser devidas às diferenças nas plataformas utilizadas e suas características peculiares, como cobertura do genoma, número de sequências e tamanho de leituras.

Beheregaray e Sunnucks (2000) identificaram 12 loci microssatélites para as espécies de peixe-rei O. argentinensis e O. perugiae, e Koshimizu et al. (2009) identificaram 17 loci SSRs para $O$. bonariensis e O. hatcheri. Ambos os estudos utilizaram a técnica de criação de bibliotecas enriquecidas para loci SSRs, clonagem, hibridização, isolamento e sequenciamento; comparando com os resultados obtidos neste estudo, a técnica utilizada por esses autores identifica um baixo número de SSRs, além de apresentar alto custo e elevado tempo de trabalho.
A repetição mais frequente encontrada foi o tetranucleotídeo (Fig. 1), sendo que Luo et al. (2012) obtiveram em "Tarim Schizothoracin” (Schizothorax biddulphi) e Lance et al. (2013) em "Speckled Dace” e "Mountain Whitefish" mais motivo dinucleotídeo.

Para tetranucleotídeos, o motivo de SSR mais frequente encontrado foi ATGG. Segundo Meglécz et al. (2012), para Chordata em geral, o motivo mais comum encontrado para tetranucleotídeo foi AGAT e, em plantas AAAT, resultados distintos em ambos os estudos e grupos de espécies, o que sugestiona a vasta variação que pode ser encontrada dentre as repetições de loci microssatélites.

Do total de PAL, foram selecionados os "Best PALs” (bPALs), estes loci SSRs apresentam unidades longas de repetições (4mers, 5mers e 6 mers) e trechos mais longos de repetições (mais de sete unidades de repetições observadas), que são mais propensos a serem variáveis nas populações de O. humensis (Castoe et al., 2012; Lance et al., 2013). O número de bPAL foi de 167 (Fig. 2), valores próximos aos encontrados por Castoe et al. (2012) em aves (100 - 450), e inferiores aos resultados de Lance et al. (2013) em peixes $(4.635-6.631)$.

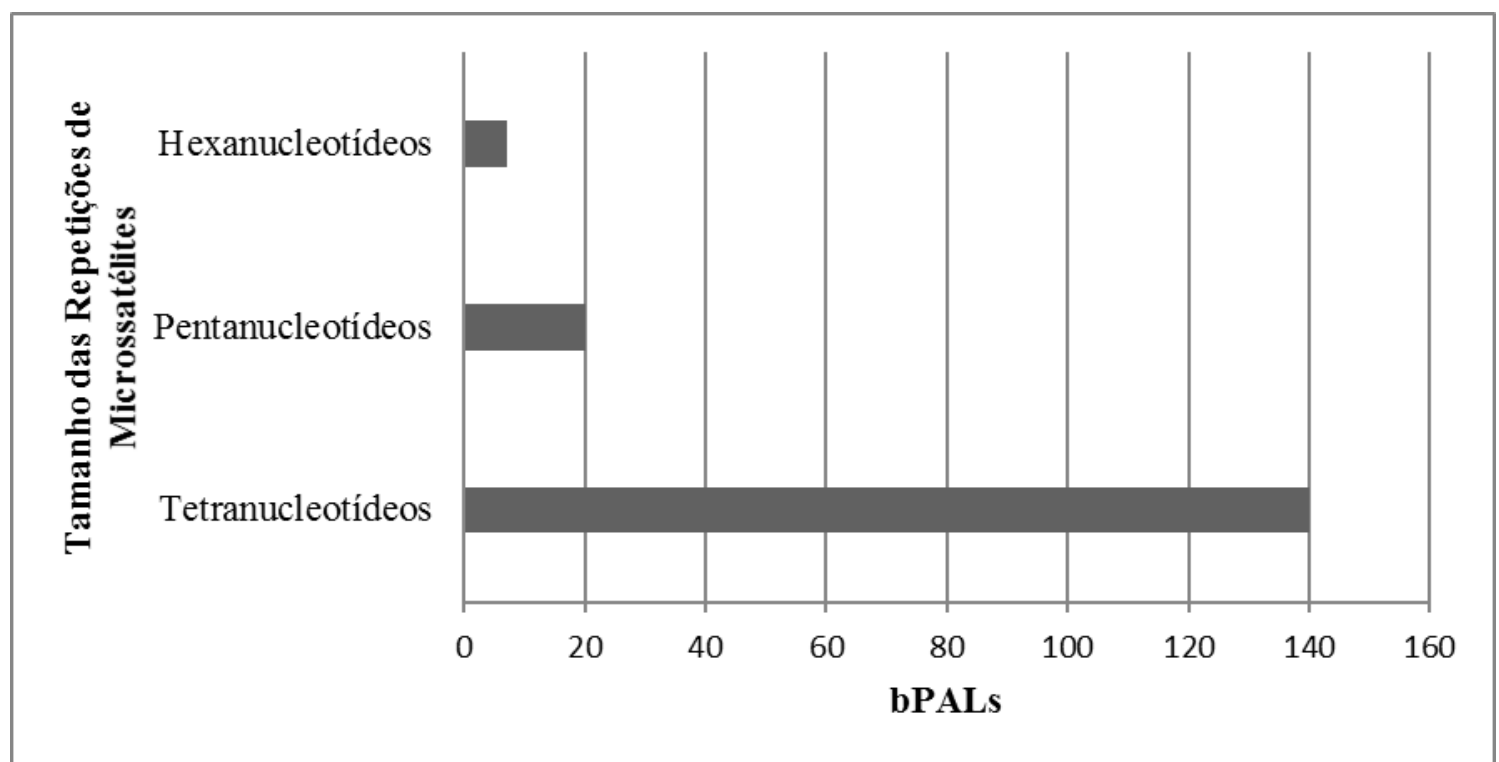

Figura 2. Loci de melhor qualidade (bPALs) identificados por tamanho das repetições de microssatélites. 
Os resultados demonstram que, com uma pequena cobertura do genoma de $O$. humensis, foi possível a identificação de um grande número de loci potencialmente amplificáveis de microssatélites. Além disso, foi gerado um número ótimo de bPAL, com potencial para análise de variabilidade genética dessa espécie.

Palavras-chave: peixe-rei, microssatélites, marcadores moleculares, PAL

\section{ABSTRACT}

In this work, 25,806 potentially amplifiable microsatellite loci (PAL) were identified in pejerrey, (Odontesthes humensis), with 21\% of dinucleotide, 22\% trinucleotide, 37\% tetranucleotide, 13\% pentanucleotide and 7\% hexanucleotide. Of the total loci, 167 were classified as "Best PAL", more likely to be variables in populations. The results show that with a small coverage of the genome it was possible to identify a large number of microsatellite loci.

Keywords: pejerrey, microsatellite, molecular marker, $P A L$

\section{AGRADECIMENTOS}

Os autores agradecem ao Conselho Nacional de Desenvolvimento Científico e Tecnológico (CNPq) por fornecer recursos para o desenvolvimento desta pesquisa.

\section{REFERÊNCIAS}

BARRERO, N.M.L.; POVH, J.A.; RIBEIRO, R.P. et al. Comparison of DNA extraction protocols of fish fin and larvae samples: modified salt $(\mathrm{NaCl})$ extraction. Cienc. Invest. Agraria, v.35, p.65-74, 2008.

BARROSO, R.M.A.; HILSDORF, W.S.; MOREIRA, H.L.M. et al. Identification and characterization of microsatellites loci in Brycon opalinus (Cuvier, 1819) (Characiforme, Characidae, Bryconiae). Mol. Ecol. Notes, v.3, p.297-298, 2003.

BEHEREGARAY, L.B.; SUNNUCKS, P. Microsatellite loci isolated from Odontesthes argentinensis and the $O$. perugiae species group and their use in other South American silverside fish. Mol. Ecology, v.9, p.629-630, 2000.

CASTOE, T.A.; POOLE, A.W.; KONING, A.P.J. et al. Rapid Microsatellite Identification from Illumina Paired-End Genomic Sequencing in Two Birds and a Snake. PLoS ONE, v.7, p.110, 2012.

DYER, B.S. Systematic revision of the South American silversides (Teleostei, Atheriniformes). Biocell, v.30, p.69-88, 2006.
GUYON, R.; RAKOTOMANGA, M.; AZZOUZI, N. et al. A high-resolution map of the Nile tilapia genome: a resource for studying cichlids and other percomorphs. BMC Genomics, v.13, p.117, 2012.

IRANAWATI, F.; JUNG, H.; CHAND, V. et al. Analysis of Genome Survey Sequences and SSR Marker Development for Siamese Mud Carp, Henicorhynchus siamensis, Using 454 Pyrosequencing. Inter. J. Molecular Sci., n.13, p.10807-10827, 2012.

JIANG, Y.; GAO, X.; SHIKAI, L. et al. Whole genome comparative analysis of channel catfish (Ictalurus punctatus) with four model fish species. BMC Genomics, v.14, p.1-11, 2013.

KATAGIRI, T.; KIDD, C.; TOMASINO, E. et al. A BAC-based physical map of the Nile tilapia genome. BMC Genomics, v.6, p.1-6, 2005.

KOSHIMIZU, E.; STRÜSSMANN, C.A.; TEJEDOR, E.D. et al. Development of polymorphic microsatellite loci for two Atherinopsid fishes, pejerrey (Odontesthes bonariensis) and Patagonian pejerrey (O. hatcheri). Mol. Ecol. Resource, v.9, p.14601466, 2009.

LANCE, S.L.; LOVE, C.N.; NUNZIATA, S.O. et al. 32 species validation of a new Illumina paired-end approach for the development of microsatellites. PLoS ONE, v.8, p.1-11, 2013.

LIEN, S.; GIDSKEHAUG, L.; MOEN, T. et al. A dense SNP-based linkage map for Atlantic salmon (Salmo salar) reveals extended chromosome homeologies and striking differences in sex-specific recombination patterns. BMC Genomics, v.12, p.1-10, 2011. 


\section{Identificação de loci...}

LUO, W.; NIE, Z.; ZHAN, F. et al. Rapid Development of Microsatellite Markers for the Endangered Fish Schizothorax biddulphi (Günther) Using Next Generation Sequencing and Cross-Species Amplification. International Journal of Molecular Science, v.13, p.1494614955, 2012.

MEGLÉCZ, E.; NÈVE, G.; BIFFIN, E.; GARDNER, M.G. Breakdown of Phylogenetic Signal: A Survey of Microsatellite Densities in 454 Shotgun Sequences from 154 Non Model Eukaryote Species. PLoS ONE, v.7, p.1-15, 2012.
ROUCHKA, E.C. Database of exact tandem repeats in the Zebrafish genome. BMC Genomics, v.11, p.1-11, 2010.

TAVARES, R.A.; NUNES, M.D.; ALMEIDA, D.B. et al. Utilization of microsatellite markers to form families of "pejerrey" Odontesthes bonariensis in a genetic breeding program. Arq. Bras. Med. Vet. Zootec., v.63, p.1263-1267, 2011. 